\title{
Clinical and Genetic Study of Friedreich's Ataxia and Ataxia with Vitamin E Deficiency in 44 Moroccan Families
}

\author{
Fatima Imounan1, Naima Bouslam1, El Hachmia Aitbenhaddou1, Wafa Regragui1, \\ Ahmed Bouhouche1, Ali Benomar',2, Mohammed Yahyaoui1

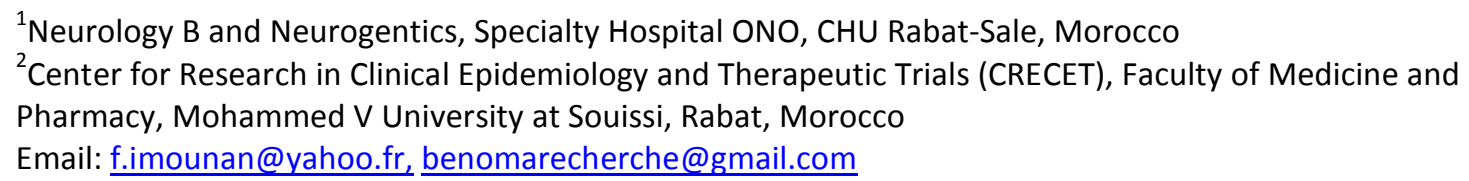

Received 29 May 2014; revised 29 June 2014; accepted 11 July 2014

Copyright (C) 2014 by authors and Scientific Research Publishing Inc.

This work is licensed under the Creative Commons Attribution International License (CC BY). http://creativecommons.org/licenses/by/4.0/

(c) (7) Open Access

\begin{abstract}
Introduction: Friedreich ataxia (FRDA) is a multi-system autosomal-recessive disease, the most common one of the genetically inherited ataxias. FRDA occurs as a consequence of mutations in the frataxin gene, with an expansion of a GAA trinucleotide. Ataxia with vitamin E deficiency (AVED) is characterized clinically by neurological symptoms with often striking resemblance to those of Friedreich's ataxia (FA) but serum concentrations of vitamin E are low. Aim of study: To study clinical and genetic features of the Friedreich's ataxia and AVED patients in 44 Moroccan families. Patients and Methods: Retrospective series of 72 Moroccan patients displaying Friedreich's ataxia syndrome was recruited over a period of 22 years (1987-2009). All patients had a clinical and ophtalmological examinations, 30 patients underwent electromyography, and CT scan was performed in 29 patients. GAA repeats in the frataxin gene and the 744 del A mutation $\alpha$-TTP gene were performed in all patients. Results: 17 patients (24\% of cases) had the 744 del A mutation in the $\alpha$-TTP gene responsible of ataxia with vitamin E deficiency (AVED) phenotype. 55 patients ( $76 \%$ of cases) had GAA expanded allele in the first intron of the frataxin gene. Phenotype-genotype correlation revealed a high frequency of head titubation, decreased visual acuity and slower disease progression in AVED than in Friedreich's ataxia phenotype $(p<0.05)$. Conclusion: Our study represents a large series which highlight the clinical and genetic differences between AVED and Friedreich's ataxia. AVED patients have a better prognosis after alpha-tocopherol treatment.
\end{abstract}

\section{Keywords}

Friedreich's Ataxia, Ataxia with Vitamin E Deficiency, GAA Expansion, 744 Del A Mutation 


\section{Introduction}

Autosomal recessive cerebellar ataxias are a heterogeneous group of rare neurological disorders involving both the central and the peripheral nervous systems and in some case other systems and organs, and characterized by degeneration or abnormal development of cerebellum and spinal cord, autosomal recessive inheritance and, in most cases, early onset occurring before the age of 20 years [1]. Friedreich ataxia (FA), an autosomal recessive neurodegenerative disorder, is the most common hereditary ataxia. In 1988, the FA locus was localized on the chromosome 9 [2]. Most mutations appeared to be unstable expansions of a GAA repeat in the first intron of the gene [3]. The disorder usually appears before adolescence and is generally characterized by uncoordinated limb movements, dysarthria, nystagmus, decreased or absent tendon reflexes, Babinski sign, decreased vibration sense, scoliosis, pes cavus and hammer toes. The diagnostic criteria were revised by Harding to include patients with onset up to the age 25 [3]. Ataxia with isolated vitamin E deficiency (AVED) is a hereditary ataxia caused by mutations in the $\alpha$-tocopherol transfer protein gene, $\alpha$-TTP. Patients with AVED show clinical signs similar to those in Friedreich ataxia, including gait and limb ataxia, dysarthria, lower limb areflexia, loss of vibration and positional sense, and bilateral Babinski sign. In contrast, cardiomyopathy and glucose intolerance are much less frequent, and head titubation and dystonia are observed in some patients only. Diagnosis is based on the finding of low serum vitamin $\mathrm{E}$ values $(<2.5 \mathrm{mg} / \mathrm{L}$; normal values $6-15 \mathrm{mg} / \mathrm{L})$ in absence of malabsorption. The 744 Del A frame shift mutation is the most frequently found defect and is distributed as a result of a founder effect in North African and southern Italian patients [1].

In this study, we report clinical, genetic features and disease progression in 55 patients of GAA expansion and 17 patients with 744 Del A mutations in 44 Moroccan families. This study represents an extension of a previous one published in 2002 which included 15 families with 29 patients.

\section{Patients and Methods}

\subsection{Clinical Evaluation}

Over a period of 1987 and 2009 to the department of neurology, of Hôpital des Spécialités, Rabat, Morocco, 252 cases of recessive ataxia were recruited. The Ataxia with GAA represented 24.6\% of cases, and AVED was present in $7.5 \%$ of cases from autosomal recessive ataxias (ARCA)

72 patients (55 patients with GAA expansion, 17 patients AVED) belonging to 44 Moroccan families displaying Friedreich's ataxia syndrome were included in our study and the other forms of autosomal recessive cerebellar ataxia were excluded.

All patients were evaluated by means of an operating record (SPATAX EUROSPA proposal on 2009 for diagnostic form for spinocerebellar degeneration).

The patients were examined through a clinical examination, mental status and a standard set of investigations including a glycemia test and a lipidogram.

All patients had ophthalmological examination. 30 patients underwent electromyography, and Computed Tomography scan was performed on 29 patients.

Five stages of disease disability were distinguished as previously described (INSERM U289, Fédération de Neurologie, Hôpital de la Salpétrière, Paris): (SPATAX group).

Stage 0: Patients at risk with normal examination results.

Stage 1: Clinical signs of FA founded at clinical exam.

Stage 2: Functional signs but able to walk unaided.

Stage 3: Clinical and functional signs, and unable to walk without help.

Stage 4: Confined to wheelchair, able to stand but not to walk.

Stage 5: Bedridden: unable to stand.

\subsection{Genetics Analysis}

All patients underwent a blood sample with the DNA extraction and genetic research analysis of GAA expansion and 744 Del A mutation.

\subsubsection{GAA Repeats}

GAA repeats in the Frataxin gene were analyzed by Southern blot, as previously described. Ten micrograms of 
genomic DNA was digested with the restriction enzyme BsiHKAI (New England Biolabs), electrophoresis on $0.9 \%$ agarose gels, blotted on HybondR $\mathrm{N}+$ membranes and hybridized with an a32P-radiolabeled 463-bp genomic probe containing exon 1 [4] of the Frataxin gene that recognizes a 2.4-kb restriction fragment. This technique allows more accurate assessment of the number of GAA repeats and a better resolution of the two alleles than the 8.2-kb restriction fragment previously used.

\subsubsection{Direct Detection of the 744 del A Mutation}

The 744 Del A mutation was detected by MboII restriction digestion of a PCR fragment containing part of exon 3 of the a-TTP gene [5]. The restriction fragments were electrophoresed on a $10 \%$ polyacrylamide gel for $4 \mathrm{~h}$, stained with ethidium bromide and visualized under UV light.

Part of exon C was amplified with the following primers: D,5'GACATTCTTCCTCTGGAATATG-3' and R,5' GATAATCTTCAGACTTCATTATAAA-3' with 30 cycles of $1 \mathrm{~min}$ at $94^{\circ} \mathrm{C}, 1 \mathrm{~min}$ at $54^{\circ} \mathrm{C}$ and $1 \mathrm{~min}$ at $72^{\circ} \mathrm{C}$.

\subsection{Statistical Analysis}

Statistical analysis was performed by SPSS.13.0. Quantitative variables were expressed as mean and standard deviation when the distribution was normal and as median and quartiles when the distribution was not Gaussian. Qualitative data were expressed as numbers and percentages. The mean frequencies of each sign in the GAA expansion and 744 Del A groups were compared with the Student's T-test and Fisher's exact test, if necessary and $\mathrm{Ki} 2$ test. $\mathrm{p}$ was significant if $\mathrm{p}<0.05$.

\section{Results}

\subsection{Genetic Characteristics}

55 of the 72 patients from 44 families with FA phenotypes had GAA expansions in the Frataxin gene. The size of the expansions varied from 1.3 to $2.5 \mathrm{~kb}$. The mean numbers of GAA repeats were $530 \pm 80$ on the smaller allele and $680 \pm 80$ repeats on the larger allele. The mean size of all expansions was $1.85 \pm 0.36 \mathrm{~kb}$.

The other 17 patients had the 744 Del mutation. The MboII restriction site was absent in all the affected patients from the AVED families, but it was found on the normal chromosomes of AVED family members.

In order to correlate disease severity with the type of mutation, the clinical features of both groups of patients were compared.

\subsection{Demographic and Clinical Characteristics of Population Study (Table 1)}

Our study is a retrospective series of 72 Friedreich's ataxia syndrom patients. The average age of our population was $36 \pm 10$ years. The average age at the exam was $21 \pm 8$ years. The age at onset was between 7 and 39 years, with mean of $15 \pm 6$ years. There was a slight male predominance (68\% versus $32 \%$ ) with a sex ratio $=2.13$.

55 patients of 33 families had FA with GAA expansion, and 17 patients of 11 families had AVED with 744 Del A mutation.

Consanguinity was documented in 23/44 families. Sixteen of the 33 families with FA were consanguineous, while seven of the eleven AVED families were consanguineous.

All patients had clinical and ophthalmological examinations. The severity of the ataxia was different: $55.6 \%$ of patients were able to walk without help while only $5.6 \%$ of cases were confined to bed. Tendon reflexes were abolished in $87.5 \%$ of cases. Lower limb weakness was present in $62.5 \%$ of cases while upper limb weakness was observed in only $18.1 \%$ of cases. Dysathria was present in $73.6 \%$ of cases. Babinski sign was present in 63 , $9 \%$ of cases. The amyotrophy was observed in $14 \%$ of cases.

Glycemia and lipidogram were normal in respectively $97 \%$ of cases and $81 \%$ of cases. CT scan was performed in 29 patients, it's was normal in 12 patients and showed cerebellum atrophy in 17 patients. ENMG was performed in 30 patients and it's showed neuropathy in $60 \%$ of cases.

\subsection{Comparison between AVED and FA Patients (Table 2)}

Comparison between the two groups included 55 FA patient with the GAA expansion and 17 AVED patients with the 744 Del A mutation (Table 2). As expected, the two mutations affected both sexes. 
Table 1. Demographic and clinical characteristics of population study.

\begin{tabular}{lc}
\hline Age (years) mean \pm SD & $36 \pm 10$ \\
Age at exam (years) mean \pm SD & $21 \pm 8$ \\
Age at onset (years) mean \pm SD & $15 \pm 6$ \\
Sex (male) & $49 / 72(68 \%)$ \\
Consanguinity & \\
- FA & $16 / 33(48.5 \%)$ \\
- AVED & $7 / 11(63.6 \%)$ \\
Gait & \\
$-\quad$ Walk without help & $40 / 72(55.6 \%)$ \\
$-\quad$ Confined to bed & $4 / 72(5.6 \%)$ \\
Abolished tendon reflexes & $63 / 72(87.5 \%)$ \\
LL weakness & $45 / 72(62.5 \%)$ \\
UL weakness & $13 / 72(18.1 \%)$ \\
Dysathria & $53 / 72(73.6 \%)$ \\
BB signs & $46 / 72(63.9 \%)$ \\
Amyotrophy & $10 / 72(14 \%)$ \\
Normal Glycemia & $36 / 37(97 \%)$ \\
Lipidogram abnormal & $7 / 37(18 \%)$ \\
CT scan & \\
$-\quad$ Normal & $13 / 29(44.8 \%)$ \\
$-\quad$ Atrophy & $16 / 29(55.1 \%)$ \\
\hline
\end{tabular}

Table 2. Comparison of Clinical characteristics.

\begin{tabular}{lccc}
\hline & FA patients GAA expansion & AVED patients 744 del A & p \\
\hline Age of onset & $15.8 \pm 6.7$ & $12.2 \pm 3.5$ & 0.047 \\
Mean age of exam & $22.2 \pm 8.5$ & $17.6 \pm 6.6$ & 0.048 \\
Babinski signs & $22 \%$ & $8 \%$ & 0.61 \\
Head titubation & $0 / 55$ & $12 / 17$ & 0.000 \\
D visual acuity & $9 / 55$ & $7 / 17$ & 0.031 \\
Gait & $54 / 55$ & $16 / 17$ & 0.2 \\
Dysarthria & $43 / 55$ & $10 / 17$ & 0.1 \\
Malformation & $80 \%$ & $76 \%$ & 0.5 \\
Amyotrophy & $70 \%$ & $30 \%$ & 0.043 \\
Severity & $24 / 55$ & $4 / 17$ & 0.45 \\
Lipidogram anormal & $3 / 27$ & $4 / 10$ & 0.1 \\
Electromyography & & & 0.47 \\
$-\quad$ Normal & $11 / 22$ & $3 / 8$ & 0.47 \\
$-\quad$ Axonal & $10 / 22$ & $4 / 8$ & 0.47 \\
$-\quad$ Demyelinating & $1 / 22$ & $1 / 8$ & 0.005 \\
CTscan cerebellum atrophy & $16 / 23$ & $0 / 6$ & \\
\hline
\end{tabular}


- We found that 38/55 FA patients and 11/17 AVED patients were male, but without significant difference (p $=0.73$ ).

- The ages of the patients ranged from 4 to 43 .

- The mean age of examination was $22.2 \pm 8.5$ for the FA patients and $17.6 \pm 6.6$ years for the AVED patients $(\mathrm{p}=0.048)$.

- The mean age at onset of the disease was $15.9 \pm 6.7$ for the FA patients and $12.2 \pm 3.5$ for the AVED patients $(p=0.047)$.

- The presenting symptom was gait ataxia in all FA patients and in 16/17 AVED patients. But there is no statistically significant difference between 2 groups with $(p=0.2)$.

- Head titubation was present at the frequency of 70\% in the AVED patients and were more frequent than in the FA patients $(\mathrm{p}=0.000)$.

- Decreased visual acuity was present at the frequency of $42 \%$ in the AVED patients and were more frequent than in the FA patients $(16.3 \%)(\mathrm{p}=0.031)$.

- In contrast the amyotrophy was more frequent in FA patients than in AVED patients. There was a statistically significant difference between 2 groups with $(\mathrm{p}=0.043)$.

- Weakness in the legs, Babinski signs, dysarthria, malformation were more frequent in FA patients than in AVED patients, but there is no statistically significant difference between 2 groups with $(p=0.2)$

- When disease severity was compared in FA and AVED patients, FA patients had more advanced disease than the AVED patients but without a significant difference between 2 groups ( $p=0.45)$.

- When patients with the same degree of handicap (stage 2) were compared, onset of the disease (FA patients: $15.8 \pm 6.7$ and AVED patients: $12.2 \pm 3.5$ ) is earlier for AVED patients. These data suggest that FA patients deteriorate more rapidly than the AVED patients.

- Fundoscopic examination revealed retinitis pigmentosa in 2/17 AVED patients but in none of the FA patients.

- Electromyography in five AVED patients revealed moderate axonal neuropathy in four and both axonal and demyelinating defects in one case. One of 22 FA patients analyzed by electromyography had severe demyelinating neuropathy. The eight others presented severe axonal neuropathy. In comparison of two groups there is no significant difference between AVED and FA patients $(p=0.7)$

- CT scans were normal in 7/23 FA patients and 6/6 AVED patients in which they were performed. However, 16 FA patients had atrophy of the cerebellum with significant difference between 2 groups $(p=0.005)$.

\section{Discussion}

In our series, AVED (11 families, 17 patients) was frequent as FA (33 families, 52 patients), although this must be confirmed in a larger series of patients (252 Moroccan patients are still under study).

In Friedreich ataxia the clinical variability in age at onset, severity and associated symptoms is due in part to variability in the size of the GAA expansion (530 - 680). This study follows the one published in 2002 and confirmed the same results [3].

In our series, this variability was observed. In all of patients with negative 744 Del A mutation of alpha-TTP gene and normal lipid absorption GAA expansions was found.

Phenotypic variability of our AVED patients, who did not fulfill all the criteria for FA [6], appeared greater than expected. All of them were homozygous for the 744 Del A mutation of the $\alpha$-TTP gene.

The symptoms in our AVED patients were more severe and earlier than in the series studied by Yokota et al., where a His101 Gln mutation might have left residual $\alpha$-TTP activity [7] [8].

Compared to Cavalier's AVED patients (28\%) [9], we observed that head titubation was far more frequent in our 744 Del A AVED patients (70\%).

Decreased visual acuity was found in $42 \%$ of AVED patients but in only 16\% of FA patients. This decreased visual acuity is related to retinitis pigmentosa. It can be the first symptom, before the onset of cerebellar ataxia, contrasting with Yokota who assumed that duration of deficiency is responsible for retinis pigmentosa phenotypic variation [8]. Decreased visual acuity was not especially caused by His101 Gln mutations in the $\alpha$ - TTP gene, but also by the 744 Del A mutation [8].

Neuropathy was also less frequent and less severe in our AVED patients than in our FA patients, in whom it was less frequent than previously described and was not related to disease duration [4]. Zouari et al. analyzed peripheral nerve conductance in 15 patients with FA and 15 patients with AVED patients. They confirmed that 
axonal neuropathy was more severe in FA than in AVED [10]. The pathological mechanisms appear therefore to differ in the two diseases.

Cardiomyopathy is the most common systemic finding but seems to be less common in AVED than in Friedreich's ataxia. The mechanism underlying this pathogenesis seems to be increased oxidative stress. Therefore, vitamin E deficiency ataxia is more severe in Moroccan patients. The Moroccan AVED presented a phenotype as severe as typical FA phenotype. Clinical diagnosis of these diseases is very difficult and needed obligatory molecular analysis [11].

The occurrence of cerebellar atrophy in our study is high compared to other studies [12]. This high incidence may be explained by the delay in diagnosis of Friedreich's ataxia.

The largest group of AVED patients is found in North Africa; they share one of the most common mutations for the $\alpha$-TTP gene (744 Del A). However, different mutations have been found among various ethnic groups in Europe, North America and Asia [8]. In a recent study, the first case of ataxia with isolated vitamin E deficiency has been identified in the Netherlands [13]. An Italian study conducted by Mariotti and coll. involved 16 patients from 12 families; they have the most common mutations 744 Del A and 513insTT, but two novel pathogenic mutations have been identified, a truncating mutation (219insAT) and a missense mutation (Gly246Arg). In spite of the development of spasticity and retinitis pigmentosa in a few patients during therapy, the vitamin $\mathrm{E}$ supplementation allowed a stabilization of the neurological conditions in most of the patients and seems to stop progression of the disease, and can mildly improve cerebellar ataxia [14]. This result suggests that a prompt genetic characterization of AVED may promote an early effective treatment of the disease.

\section{Conclusion}

Our study represents a large series, which highlights the clinical differences between AVED and Friedreich's ataxia. AVED resulting from the 744 Del A mutation is frequent in Morocco. It is characterized by frequent head titubation, decreased visual acuity, less frequent neuropathy and slower disease progression and AVED patients have a better prognosis after alpha-tocopherol treatment than Friedreich's ataxia patients.

\section{Disclosure of Interest}

The authors declare that they have no conflicts of interest concerning this article.

\section{References}

[1] Palau, F. and Espinós, C. (2006) Autosomal Recessive Cerebellar Ataxias Review. Orphanet Journal of Rare Diseases, 1, 47. http://dx.doi.org/10.1186/1750-1172-1-47

[2] Chamberlain, S., Shaw, J., Rowland, A., et al. (1988) Mapping of Mutation Causing Friedreich's Ataxia to Human Chromosome 9. Nature, 334, 248-250. http://dx.doi.org/10.1038/334248a0

[3] Benomar, A., Yahyaoui, M., Meggouh, F., Bouhouche, A., Schmitt, M., et al. (2002) Clinical Comparison between AVED Patients with 744 Del A Mutation and Friedreich Ataxia with GAA Expansion in 15 Moroccan Families. Journal of the Neurological Sciences, 198, 25-29. http://dx.doi.org/10.1016/S0022-510X(02)00057-6

[4] Durr, A., Cossee, M., Agid, Y., et al. (1996) Clinical and Genetic Abnormalities in Patients with Friedreich’s Ataxia. The New England Journal of Medicine, 335, 1169-1175. http://dx.doi.org/10.1056/NEJM199610173351601

[5] Ouahchi, K., Arita, M., Kayden, H., et al. (1995) Ataxia with Isolated Vitamin E Deficiency Is Caused by Mutations in the a-Tocopherol Transfer Protein. Nature Genetics, 9, 141-145. http://dx.doi.org/10.1038/ng0295-141

[6] Harding, A.E. (1993) Clinical Features and Classification of Inherited Ataxias. Advances in Neurology. Raven Press, New York, 1-14.

[7] Yokota, T., Shiojiri, T., Goloda, T., et al. (1996) Retinitis Pigmentosa and Ataxia Caused by Mutation in the Gene for the $\alpha$-Tocopherol-Transfer Protein. The New England Journal of Medicine, 23, 1770-1771. http://dx.doi.org/10.1056/NEJM199612053352315

[8] Yokota, T., Shiojiri, T., Gotod, T., et al. (1997) Friedreich-Like Ataxia with Retinitis Pigmentosa Caused by the His101 Gln Mutation of the a Tocopherol Transfer Protein Gene. Annals of Neurology, 41, 826-832. http://dx.doi.org/10.1002/ana.410410621

[9] Cavalier, L., Ouahchi, K., Kayden, J.H., et al. (1998) Ataxia with Isolated Vitamin E Deficiency: Heterogeneity of Mutations and Phenotypic Variability in a Large Number of Families. The American Journal of Human Genetics, 62, 301-310. http://dx.doi.org/10.1086/301699 
[10] Zouari, M., Feki, M., Benhmida, C., et al. (1998) Electrophysiology and Nerve Biopsy: Comparative Study in Friedreich's Ataxia and Friedreich Ataxia Phenotype with Vitamin E Deficiency. Neuromuscular Disorders, 8, 416-425. http://dx.doi.org/10.1016/S0960-8966(98)00051-0

[11] Marzouki, N., Benomar, A., Yahyaoui, M., et al. (2005) Vitamin E Deficiency Ataxia with (744 Del A) Mutation on a-TTP Gene: Genetic and Clinical Peculiarities in Moroccan Patients. European Journal of Medical Genetics, 48, 2128. http://dx.doi.org/10.1016/j.ejmg.2005.01.014

[12] Mascalchi, M. (2013) The Cerebellum Looks Normal in Friedreich Ataxia. American Journal of Neuroradiology, 34, E22. http://dx.doi.org/10.3174/ajnr.A3480

[13] Ponten, S.C., Kwee, M.L., Wolters, E.C. and Zijlmans, J.C. (2007) First Case of Ataxia with Isolated Vitamin E Deficiency in the Netherlands. Parkinsonism \& Related Disorders, 13, 315-316. http://dx.doi.org/10.1016/j.parkreldis.2006.06.011

[14] Mariotti, C., et al. (2004) Ataxia with Isolated Vitamin E Deficiency: Neurological Phenotype, Clinical Follow-Up and Novel Mutations in TTPA Gene in Italian Families. Neurological Sciences, 25, 130-137. http://dx.doi.org/10.1007/s10072-004-0246-z 
Scientific Research Publishing (SCIRP) is one of the largest Open Access journal publishers. It is currently publishing more than 200 open access, online, peer-reviewed journals covering a wide range of academic disciplines. SCIRP serves the worldwide academic communities and contributes to the progress and application of science with its publication.

Other selected journals from SCIRP are listed as below. Submit your manuscript to us via either submit@scirp.org or Online Submission Portal.
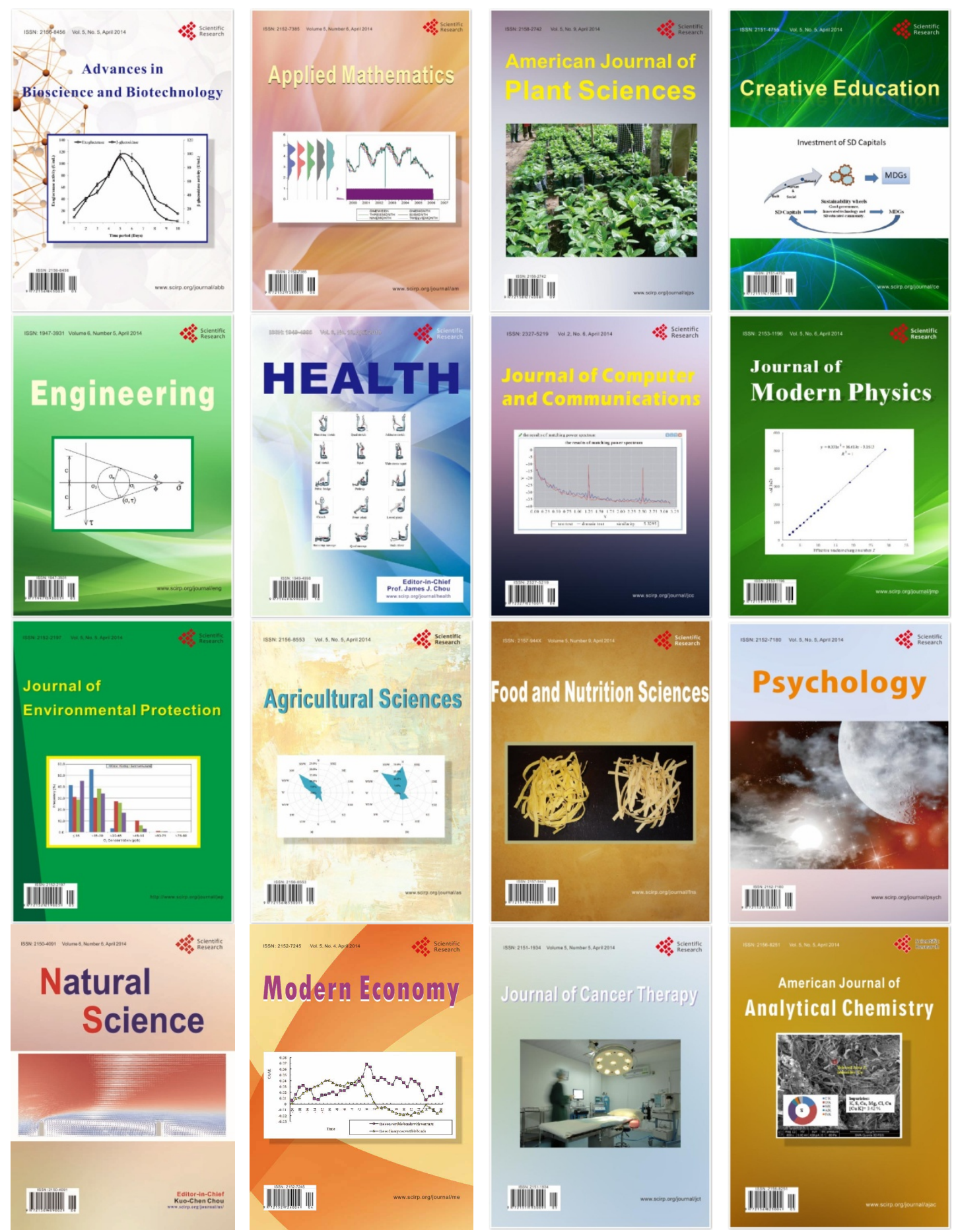